\title{
Evaluation of Dental Implants Following Ridge Preservation in Molar Extraction Sockets Affected by Advanced Periodontitis: A 30-Month Postloading Case Series
}

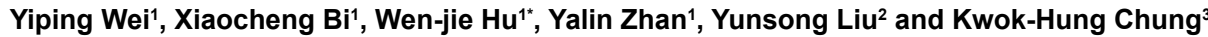

${ }^{1}$ Department of Periodontology, Peking University School and Hospital of Stomatology, National Engineering Laboratory for Digital and Material Technology of Stomatology, Beijing Key Laboratory of Digital Stomatology, Beijing, China

${ }^{2}$ Department of Restorative Dentistry, Peking University School and Hospital of Stomatology, National Engineering Laboratory for Digital and Material Technology of Stomatology, Beijing Key Laboratory of Digital Stomatology, Beijing, China

${ }^{3}$ Department of Restorative Dentistry, University of Washington, Seattle, Washington, USA

\begin{abstract}
The aim of this study is to observe the clinical efficacy of implant treatment following alveolar ridge preservation in molar extraction sockets affected by advanced periodontitis. Three patients underwent implant placement following alveolar ridge preservation in molar sockets with advanced periodontitis. During a follow-up period of 30 months, each implant was examined for the following parameters: probing depth, bleeding on probing, modified plaque index and width of keratinized tissue. Parallel periapical radiographs were taken to evaluate peri- implant marginal bone loss. Cone beam computed tomography scans were performed immediately after extraction, before implant placement, and 30 months after implant loading to assess alterations in alveolar dimensions. After ridge preservation, the average ridge width measured at $1 \mathrm{~mm}$ apically from the initial buccal or lingual crestal bone increased by $8.69 \mathrm{~mm}$. Three implants all survived and were successful. Radiological evaluation revealed an average marginal bone loss of $0.43 \mathrm{~mm}$ (range: 0.25 $0.6 \mathrm{~mm}$ ) at 1 year post loading and up to $0.51 \mathrm{~mm}$ (range: $0.33-0.75 \mathrm{~mm}$ ) after 30-month follow-up. Despite advanced periodontitis, affected molar extraction sites can be preserved and rehabilitated with implant-supported crowns. This safe and predictable treatment protocol exhibits satisfactory clinical results even after 30 months.
\end{abstract}

Keywords: Bone grafting; Marginal bone loss; Implant survival and success

\section{Abbreviations}

BOP: Bleeding on Probing; СBCT: Cone Beam Computed Tomography; DBBM: Deproteinized Bovine Bone Mineral; DICOM: Digital Imaging and Communications in Medicine; ISQ: Implant Stability Quotient; KT: Keratinized Tissue; MBL: Marginal Bone Loss ; PI: Modified Plaque Index; PPD: Peri-Implant Probing Depth

\section{Introduction}

Periodontal disease, which is seriously damaging to human health, is the main cause of tooth loss in adults [1]. Once affected by advanced periodontal disease, molars are expected to experience inferior treatment outcomes and poor prognosis in comparison with singlerooted teeth, due to anatomic factors such as presence of furcation, concavities on the root surfaces and multiple root prominence areas. Inflammation resulting from advanced periodontal disease is often not easy to control, and extraction is frequently recommended to achieve improved treatment outcomes.

However, loss of a natural tooth inevitably results in significant three-dimensional bone remodeling of the alveolar ridge. In humans, dimensional alterations have been reported to cause a ridge width reduction of up to $50 \%$ during the first year following tooth loss in premolar and molar sites, and the majority of this loss is observed within the first 3 months [2]. Studies of periodontally compromised extraction sockets are limited, but available literature shows that resorption of the residual alveolar bone in extraction sockets is more pronounced in cases for which extracted teeth are affected by periodontal disease [3-5].

The amount of hard tissue resorption due to advanced periodontitis and tooth extraction often complicates implant placement in the ideal prosthetic position. In this situation, ridge augmentation procedures prior to or simultaneously with implant placement are required to allow a restoratively driven implant placement. Alveolar ridge preservation was previously developed as a therapy to minimize remodeling and to preserve alveolar bone after tooth extraction [6]. Sisti et al. used conebeam computed tomographic measurements to study extraction socket changes where socket preservation techniques were applied at nonmolar sites exhibiting buccal bone defects of more than $5 \mathrm{~mm}$ [7]. Their results indicated that preservation procedures minimized alveolar crest resorption and provided better results in terms of bone regeneration, with bone width increasing by $8.01 \mathrm{~mm}$ compared with traditional guided bone regeneration procedures performed at the time of implant placement in untreated sites.

However an outcome more valuable than dimensional changes following ridge preservation alone would be the long-term functional success of the implant. A limited number of studies have reported the clinical and radiographic outcomes of implant placement at ridge-preserved sites. In a clinical study with a 12-month follow up, 24 implants placed in premolar or molar sites preserved with bovine bone mineral plus collagen membrane had a survival rate of $100 \%$ and a success rate of $95.83 \%$ [8]. Tallarico et al. evaluated the outcomes of 12 molar extraction sites treated with implants following ridge preservation. Their findings showed that mean marginal bone loss was

${ }^{*}$ Corresponding author: Wenjie Hu, DDS, PhD, Professor and Vice Chairman Department of Periodontology, Peking University School and Hospital of Stomatology National Engineering Laboratory for Digital and Material Technology of Stomatology, Beijing Key Laboratory of Digital Stomatology, Tel: 86-82195368; Fax: 86-01062173402; E-mail: huwenjie@pkuss.bjmu.edu.cn

Received July 30, 2018; Accepted August 07, 2018; Published August 10, 2018

Citation: Wei Y, Bi X, Hu W, Zhan Y, Liu Y, et al. (2018) Evaluation of Denta Implants Following Ridge Preservation in Molar Extraction Sockets Affected by Advanced Periodontitis: A 30-Month Postloading Case Series. Dentistry 8: 505. doi:10.4172/2161-1122.1000505

Copyright: ( 2018 Wei Y, et al. This is an open-access article distributed unde the terms of the Creative Commons Attribution License, which permits unrestricted use, distribution, and reproduction in any medium, provided the original author and source are credited. 
$0.23 \pm 0.06 \mathrm{~mm} 1$ year after loading, which was significantly lower than that for immediate implants with grafting [9]. All the above results were based on sockets with three walls intact and at least $80 \%$ of four walls intact. To our knowledge, no studies have been published reporting outcomes of dental implants after the healing of preserved molar extraction site with advanced periodontal disease. The aim of this case series was therefore to assess the clinical efficacy of implant treatment following alveolar ridge preservation in the case of molar extraction sockets affected by advanced periodontitis.

\section{Materials and Methods}

\section{Study design and participants}

Three patients with molar extraction sockets affected by advanced periodontitis and who planned to have subsequent implant-supported rehabilitation were initially recruited for a prospective clinical trial involving ridge preservation [10]. This case series was conducted in accordance with the World Medical Association Declaration of Helsinki and approved by local ethics committee. Written informed consent was obtained from all participants following the guidelines of the committee for the research process. The inclusion and exclusion criteria have been previously described in detail.

\section{Surgical and clinical procedures}

All patients underwent initial periodontal therapy in order to provide a more favorable oral environment for wound healing. The ridge preservation protocol is described in detail elsewhere [10]. In summary, the hopeless tooth was extracted atraumatically, sectioning the roots with diamond fissure burs and allowing root separation within the socket if required to preserve the surrounding soft and hard tissues (Figure 1). For each patient, a full thickness mucoperiosteal flap was raised utilizing vertical releasing incisions. The alveolus was filled with deproteinized bovine bone mineral (DBBM, Geistlich) and covered by a bioabsorbable porcine collagen membrane (Bio-Gide, Geistlich). The flap was then coronally repositioned to cover as much of the filled material as possible. Cone beam computed tomography (CBCT) scans were taken immediately after ridge preservation, which were taken at a slice thickness of $0.125 \mathrm{~mm}$ and field of view size $8^{\star} 8$ centimeters for 360 degree with the same imaging unit (NewTom VG, Aperio Services, Italy) and pixel size of $0.125 \mathrm{~mm}$; exposure parameters were: $15 \mathrm{~s}, 110$ $\mathrm{kVp}, 12-17 \mathrm{mAs}$.

6 months after the ridge preservation procedure, a second CBCT scan was obtained before implant surgery. The two sets of DICOM (Digital Imaging and Communications in Medicine) data were transferred to a volumetric imaging software (Mimics 17.0, Materialise, Leuven, Belgium) in which an investigator superimposed the CBCTs and performed measurements to determine bone volume changes between the time of extraction (baseline) and 6 months following ridge preservation.
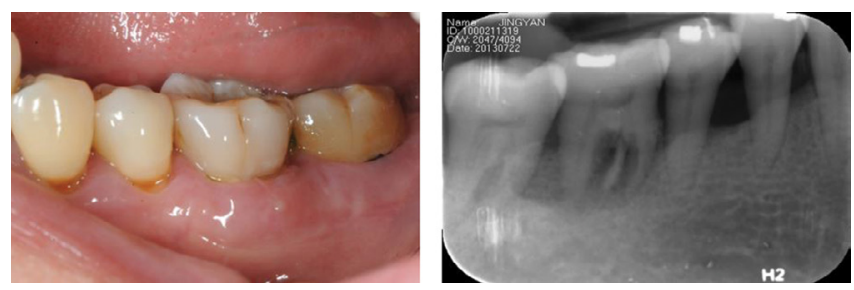

Figure 1: The tooth at position \#30 with advanced periodontitis: a representative case scheduled for extraction.
By means of intracrevicular incisions minimally extended to the neighbouring teeth, a full-thickness mucoperiosteal flap was elevated 3-4 $\mathrm{mm}$ from the buccal/lingual bone crest. A trephine $(2.8 \times 6.0 \mathrm{~mm})$ was used with copious chilled saline irrigation to remove a core from the center of the alveolar crest, which was then carefully removed from the trephine using a periodontal probe. Cores were fixed in $4 \%$ formalin. An osteotomy was performed for the placement of a dental implant (Straumann AG, Basel, Switzerland). Classification of bone quality was assessed during surgery according to Lekholm and Zarb [11]. Insertion torque and implant stability quotient (ISQ) values were recorded. All surgical procedures were performed by the same experienced operator. Bone samples were decalcified, sectioned and prepared for histological and histomophometric analysis using toluidine blue staining.

Implant fixtures were restored 6 months post-implant placement, at which point the implant was loaded. Implant crowns were screwretained. All restorative treatment was performed by the same experienced operator. The treatment phase of one of the patients is presented in Figure 2.

\section{Outcome measures and follow-up}

Clinical examination: Patients were clinically evaluated for the first time at the moment of implant loading, and then every 6 months thereafter. All clinical examinations were conducted by the same clinician, who previously had been calibrated for consistency of periodontal (peri-implant) probing technique. During each examination, the following clinical parameters were recorded for all implants: peri-implant probing depth (PPD), bleeding on probing (BOP), modified plaque index (PI) [12] and width of keratinized tissue (KT).

PPD was measured at six sites (mesio buccal, mid buccal, disto buccal, disto lingual, mid lingual and mesio lingual) per implant, and was measured to the nearest millimeter using a graded probe $(\mathrm{Hu}-$ Friedy, Chicago, IL). BOP and PI were measured at two sites (midbuccal and mid-lingual).
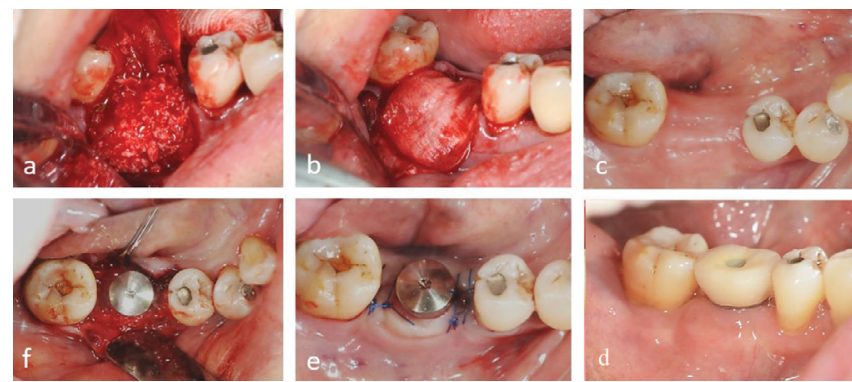

Figure 2: The treatment phase of a representative case. (a) Following atraumatic extraction the ridge is preserved with DBBM; (b) The collagen membrane was covered; (c) Healing after 6 months following ridge preservation; (d,e) Implan placed in the preserved site; (f) The final crown at the implant in position \#30 has been successfully placed.

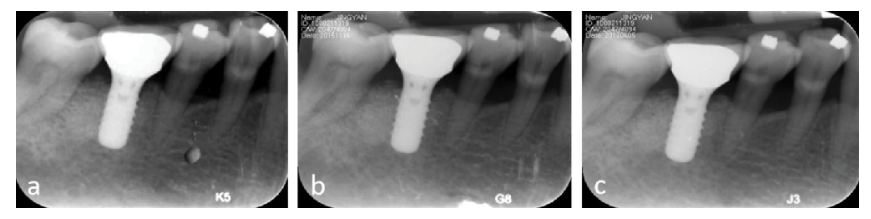

Figure 3: Periapical radiograph (a) Immediately after implant placement; (b) At 1-year follow up; (c) At 30-month follow-up. 
Citation: Wei Y, Bi X, Hu W, Zhan Y, Liu Y, et al. (2018) Evaluation of Dental Implants Following Ridge Preservation in Molar Extraction Sockets Affected by Advanced Periodontitis: A 30-Month Postloading Case Series. Dentistry 8: 505. doi:10.4172/2161-1122.1000505

\begin{tabular}{|c|c|c|c|c|c|c|}
\hline Patient & Age & Gender & Site & $\begin{array}{c}\text { Implant Type } \\
\text { Insertion Torque (Ncm) ISQ }\end{array}$ & $\begin{array}{c}\text { Bone } \\
\text { Quality }\end{array}$ & $\begin{array}{c}\text { Primary } \\
\text { Stability }\end{array}$ \\
\hline A & 56 & Female & $\# 30$ & $\begin{array}{c}\text { Straumann 4.8 mm×10 mm } \\
\text { WN SP III }\end{array}$ & 30 & 58 \\
\hline B & 55 & Female & $\# 30$ & $\begin{array}{c}\text { Straumann 4.8 mm×10 mm } \\
\text { WN S III }\end{array}$ & 35 & 60 \\
\hline C & 46 & Male & $\# 31$ & $\begin{array}{c}\text { Straumann 4.8 mm×12 mm } \\
\text { WN SP IV }\end{array}$ & 30 & 40 \\
\hline
\end{tabular}

Table 1: Demographic data of included patients and implant sites.

\begin{tabular}{|c|c|c|c|c|c|c|}
\hline S.No. & \multicolumn{2}{|c|}{ Baseline } & \multicolumn{2}{c|}{ 6 months } & \multicolumn{2}{c|}{ Changes } \\
\hline & $\begin{array}{c}\text { Height(Buccal/ } \\
\text { lingual) }\end{array}$ & Width & $\begin{array}{c}\text { Height (Buccal/ } \\
\text { lingual) }\end{array}$ & Width & Height & Width \\
\hline A & $2.01 / 5.72$ & 1.46 & $6.76 / 4.57$ & 11.12 & $+4.75 /-1.15$ & +9.66 \\
\hline B & $3.02 / 5.82$ & 1.87 & $6.45 / 5.09$ & 12.06 & $+3.43 /-0.73$ & +10.19 \\
\hline C & $8.52 / 3.65$ & 1.36 & $7.17 / 2.7$ & 7.59 & $-1.35 /-0.95$ & +6.23 \\
\hline
\end{tabular}

Table 2: Alveolar dimensions at extraction site baseline and 6 months after ridge preservation $(\mathrm{mm})$

\begin{tabular}{|c|c|c|}
\hline Patient & 1 year & 30 months \\
\hline A & 0.25 & 0.33 \\
\hline B & 0.44 & 0.45 \\
\hline C & 0.6 & 0.75 \\
\hline
\end{tabular}

Table 3: Marginal bone loss $(\mathrm{mm})$ at implant site 1 year and 30 months after loading.

\section{Radiographic Examination}

Every 6 months after implant loading, standardized digital intraoral radiographs using an occlusal bite index were taken with paralleling technique for each implant (Figure 3). Marginal bone level, defined as the distance from the most coronal bone-to-implant contact at the mesial and distal aspect to the shoulder of the implant was measured. This was compared to a baseline periapical radiograph taken straight after insertion of the implant prosthesis, to determine the amount of marginal bone loss (MBL). For each implant, mean values of mesial and distal measurements were averaged.

After loading for 30 months, CBCT scanning was repeated. The procedure given above was used to assess the buccal and lingual contours of the alveolar bone.

\section{Criteria for Implant Survival and Success}

Thirty months after loading, implants were evaluated for survival and success. A surviving dental implant was defined as any dental implant that still in situ without mobility. The dental implant was considered to be successful if it complied with the following criteria [13]: immobile; absence of persistent signs and symptoms (pain, foreign body sensation, dysaesthesia); no $P P D>5 \mathrm{~mm}$; no $P P D=5 \mathrm{~mm}$ and BOP; a radiograph did not demonstrate continuous radiolucency around implant; after the first year of service, annual vertical bone loss not exceeding $0.2 \mathrm{~mm}$.

\section{Results}

All treatments were performed according to established clinical protocols. No post-operative complications were recorded at any included site. Three patients completed the study and attended the 30-month follow-up. Each patient contributed a single mandibular site to the study. Patients' demographic data and implant details are reported in Table 1 .

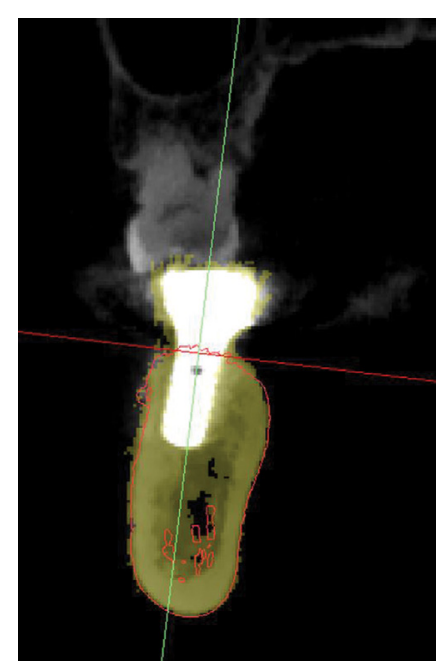

Figure 4: Buccal-lingual view and superimposition of СBCT images of a representative case before implant placement (red lines) and post-loading for 30 months.

Table 2 shows the alveolar dimensions at the time of extraction (baseline) and 6 months after ridge preservation. The average ridge width measured at $1 \mathrm{~mm}$ apically from the initial buccal or lingual crestal bone increased by $8.69 \mathrm{~mm}$ (range: $6.23-10.19 \mathrm{~mm}$ ). At the moment of implant placement, available bone heights were all more than $10 \mathrm{~mm}$ and widths were all more than $8 \mathrm{~mm}$. The histomorphometric results showed that the new bone occupied 11.54\% (range: 5.35\%-15.05\%) of the total area, the connective tissue were $53.42 \%$ (range: $34.08 \%$ $54.81 \%$ ) and the residual graft particles were $35.04 \%$ (range: $35.84 \%$ $50.87 \%)$.

No further bone augmentation was advanced at the time of implant placement. At implant placement, insertion torque was more than 30 $\mathrm{Ncm}$ for all implants. The average ISQ value was approximately 53.

Three patients all presented with low levels of plaque accumulation $(\mathrm{PI} \leq 1)$ and good gingival health with no bleeding on probing during 30-month follow-up. Peri-implant probing depths were all no more than $5 \mathrm{~mm}$, and remained relatively stable throughout 30 months with changes within $1 \mathrm{~mm}$. All sites exhibited a minimum width of $4 \mathrm{~mm}$ of keratinized tissue.

Radiological evaluation showed an average MBL of $0.43 \mathrm{~mm}$ (range: $0.25-0.6 \mathrm{~mm}$ ) at 1 year post loading and up to $0.51 \mathrm{~mm}$ (range: 0.33-0.75 mm) after 30-month follow-up (Table 3). All three implants satisfied the criteria for successful outcome.

Superimposition of CBCT images of a representative case before implant placement and after post-loading for 30 months is shown in Figure 4.

\section{Discussion}

Many clinical studies have evaluated fresh extraction sockets following tooth removal due to caries or fractures. Most of these studies evaluated single-rooted tooth sites. However, teeth are more frequently extracted due to advanced periodontitis than due to caries or fractures, and molar sites present a more complicated situation than do singlerooted tooth sites.

The aim of this case series was to evaluate radiographically and clinically the 30-month outcome of implants placed at advanced 
Citation: Wei Y, Bi X, Hu W, Zhan Y, Liu Y, et al. (2018) Evaluation of Dental Implants Following Ridge Preservation in Molar Extraction Sockets Affected by Advanced Periodontitis: A 30-Month Postloading Case Series. Dentistry 8: 505. doi:10.4172/2161-1122.1000505

periodontitis-affected molar extraction sites which had previously undergone ridge preservation with DBBM.

Ridge preservation has been proposed with the aim of preserving/ reconstructing the alveolar ridge volume. This aim is particularly relevant when implant-supported rehabilitation has been planned, because successful outcome for implant prostheses is related to best bone availability. Our results demonstrated that ridge width (of all three sites) and ridge height (of two sites) both increased after ridge preservation. The histomorphometric analysis showed that small amounts of new bone (5.35\%-15.05\% of the total area) were formed among the DBBM particles, which is not consistent with the findings of previous studies. Gholami et al. [14] evaluated the histomorphometric outcomes of socket preservation using DBBM with a collagen membrane in 14 non-molar extracted sites. Their findings revealed an increase of $27.35 \pm 12.39 \%$ vital bone. The reason for the discrepancy between these studies most likely relates to the original reasons for tooth extraction and the baseline alveolar bone defect. Despite this, alveolar ridge preservation with DBBM and collagen barrier at periodontally compromised molar sockets appears safe following thorough removal of the infectious source, as confirmed by Kim [15], and is effective and predictable for the creation of favorable conditions for implant installation.

Primary stability is affected by the quantity and quality of the available bone at implant placement [16]. The IT measures the rotational stability while the ISQ measures the axial stability of the implant. Although the ISQ values in placement of three implants placed in this study were not very high, all implants had achieved good osseointegration by the time of loading.

PI, BOP and PPD should be carefully monitored as indicators of a concrete risk of implant loss. During the 30-month follow-up, three implants all presented good gingival health. However, patients with a history of periodontitis showed higher PPDs of implants compared to periodontally healthy patients in the long term [17]. Therefore, a good periodontal maintenance program is an indispensable component of successful implant treatment.

Marginal bone loss is considered an important indicator of the health of the tissue surrounding implants. In the present case series, the 1-year peri-implant average MBL was $0.43 \mathrm{~mm}$ and the 30 -month peri-implant average MBL was $0.51 \mathrm{~mm}$. Comparing the results of the present study with those of other studies is difficult because of the low numbers, but the 30-month results confirmed that implants placed in ridge-preserved molar sites were successful. CBCT scan images revealed no significant changes of buccal and lingual bone contours (Figure 4).

Molar extraction sites affected by advanced periodontitis can be preserved and rehabilitated with implant-supported crowns using traditional procedures. The associated treatment protocol has proven to be safe and predictable, and exhibits satisfactory clinical results including continued stability after 30 months, even in the presence of advanced periodontal disease. Although this study should be replicated with a longer follow up period and a larger patient sample, these preliminary results encourage further investigation of this technique and of the use of these materials.

\section{Acknowledgement}

Study supported in part by the Capital foundation for Clinical Characteristics and Application Research, Grant \#Z161100000516042 and the Capital Medical Development and Research Fund, PRC (2011-4025-04). We thank Dr. Hollie Walsh, $\mathrm{PhD}$, for editorial assistance with the manuscript.

Note: Yiping Wei and Xiaocheng Bi contributed equally to this case report.

\section{References}

1. Frencken JE, Sharma P, Stenhouse L, Green D, Laverty D, et al. (2017) Global epidemiology of dental caries and severe periodontitis - a comprehensive review. J Clin Periodontol 44: S94-S105.

2. Schropp L, Wenzel A, Kostopoulos L, Karring T (2003) Bone healing changes and soft tissue contour changes following single-tooth extraction: a clinical and radiographic 12-month prospective study. Int J Periodontics Restorative Dent 23: 313-323

3. Ahn JJ, Shin HI (2008) Bone tissue formation in extraction sockets from sites with advanced periodontal disease: a histomorphometric study in humans. Int $J$ Oral Maxillofac Implants 23: 1133-1138.

4. Nevins M, Camelo M, De Paoli S, Friedland B, Schenk RK, et al. (2006) A study of the fate of the buccal wall of extraction sockets of teeth with prominent roots. Int J Periodontics Restorative Dent 26: 19-29.

5. Johnson K (1963) A study of the dimensional changes occurring in the maxilla after tooth extraction. Part I. Normal healing. Aust Dent J 8: 428-433.

6. Darby I, Chen ST, De Poi R (2008) Ridge preservation: what is it and when should it be considered? Aust Dent J 53: 11-21.

7. Sisti A, Canullo L, Mottola MP, Covani U, Barone A, et al. (2012) Clinical evaluation of a ridge augmentation procedure for the severely resorbed alveolar socket: multi-center randomized controlled trial, preliminary results. Clin Oral Implants Res 23: 526-535.

8. Cardaropoli D, Tamagnone L, Roffredo A, Gaveglio L (2015) Evaluation of dental implants placed in preserved and nonpreserved postextraction ridges: A 12-month postloading study. Int J Periodontics Restorative Dent 35: 677-685.

9. Tallarico M, Xhanari E, Pisano M, Gatti F, Meloni SM (2017) Molar replacemen with $7 \mathrm{~mm}$-wide diameter implants: to place the implant immediately or to wait 4 months after socket preservation? 1 year after loading results from a randomised controlled trial. Eur J Oral Implantol 10: 169-178.

10. Zhao L, Xu T, Hu W, Chung KH (2018) Preservation and augmentation of mola extraction sites affected by severe bone defect due to advanced periodontitis: A prospective clinical trial. Clin Implant Dent Relat Res 20: 333-334

11. Lekholm U, Zarb GA (1985) Patient selection and preparation. In: Branemark PI, Zarb GA, Albrektsson T. Tissue-Integrated Prostheses. Chicago: Quintessence. 199-209.

12. Mombelli A, Van Oosten MA, Schurch E Jr, Lang NP (1987) The microbiota associated with successful or failing osseointegrated titanium implants. Oral Microbiol Immunol 2: 145-151.

13. Karoussis IK, Bragger U, Salvi GE, Burgin W, Lang NP (2004) Effect of implant design on survival and success rates of titanium oral implants: a 10year prospective cohort study of the ITIs Dental Implant System. Clinical Oral Implants Research 15: 8-17.

14. Gholami GA, Najafi B, Mashhadiabbas F, Goetz W, Najafi S (2012) Clinical histologic and histomorphometric evaluation of socket preservation using a synthetic nanocrystalline hydroxyapatite in comparison with a bovine xenograft: a randomized clinical trial. Clin Oral Implants Res 23: 1198-1204.

15. Kim JJ, Ben Amara H, Schwarz F, Kim HY, Lee JW, et al. (2017) Is Ridge Preservation/Augmentation at Periodontally Compromised Extraction Sockets Safe? A Retrospective Study. J Clin Periodontol 44: 1051-1058.

16. Meredith N (1998) Assessment of implant stability as a prognostic determinant Int J Prosthodont 11: 491-501.

17. Roccuzzo M, De Angelis N, Bonino L, Aglietta M (2010) Ten-year results of a three-arm prospective cohort study on implants in periodontally compromised patients. Part 1: implant loss and radiographic bone loss. Clin Oral Implants Res 21: 490-496. 